\title{
Preparation, characterization, and evaluation of amphotericin B-loaded MPEG-PCL-g-PEI micelles for local treatment of oral Candida albicans
}

This article was published in the following Dove Press journal:

International Journal of Nanomedicine

6 June 2017

Number of times this article has been viewed

\author{
Li Zhou',* \\ Peipei Zhangl,* \\ Zhuo Chen' \\ Shaona $\mathrm{Cai}^{2}$ \\ Ting Jing ${ }^{2}$ \\ Huihui Fan² \\ Fei $\mathrm{Mo}^{2}$ \\ Jiye Zhang ${ }^{2}$ \\ Rong Lin'
}

'Department of Pharmacology, ${ }^{2}$ School of Pharmacy, Health Science Center, Xi'an Jiaotong University, Xi'an, People's Republic of China

*These authors contributed equally to this work
Correspondence: Jiye Zhang; Rong Lin Health Science Center, Xi'an Jiaotong University, No 76 Yanta Westroad, Xi'an 71006I, People's Republic of China $\mathrm{Tel} / \mathrm{fax}+862982657833$ Email zjy20I I@mail.xjtu.edu.cn; linrong029@I26.com
Abstract: Fatal Candida albicans infections in the mucosal system can occur in association with immune-compromised diseases and dysbacteriosis. Currently, amphotericin B (AmB) is considered to be the most effective antibiotic in the treatment of $C$. albicans infections, but its clinical application is limited by side effects and poor bioavailability. In order to use AmB in the local treatment of oral C. albicans infections, AmB/MPEG-PCL-g-PEI (monomethoxy poly(ethylene glycol)-poly(epsilon-caprolactone)-graft-polyethylenimine, MPP) micelles were prepared. A series of characterizations were performed. The micelles allowed a sustained in vitro release in both normal oral conditions ( $\mathrm{pH}$ 6.8) and C. albicans infection conditions ( $\mathrm{pH}$ 5.8). Then, buccal tablets containing freeze-dried powder of AmB/MPP micelles were produced by direct compression process and evaluated as regards to weight variation, hardness, and friability. In vitro drug release of the buccal tablets was measured in both the United States Pharmacopeia dissolution apparatus and the dissolution rate test apparatus, which was previously designed for simulating in vivo conditions of the oral cavity. The buccal tablets could sustainably release within $8 \mathrm{~h}$ and meet the antifungal requirements. Regarding safety assessment of AmB/MPP micelles, in vivo histopathological data showed no irritation toward buccal mucosa of the rats in both optical microscopy and ultrastructure observation of the tissues. MTT experiment proved that $\mathrm{AmB} / \mathrm{MPP}$ micelles reduced the cytotoxicity of AmB. The micelles delivered through the gastrointestinal route were also found to be non-systemic toxicity by liquid chromatography-mass spectrometry analysis. Furthermore, the antifungal action of AmB/MPP micelles was evaluated. Although AmB/MPP had no obvious improvement as compared to AmB alone in the antifungal effect on planktonic $C$. albicans, the micelles significantly enhanced the antifungal activity against the biofilm state of $C$. albicans. Thus, it was concluded that AmB/MPP micelles represent a promising novel drug delivery system for the local treatment of oral C. albicans infections.

Keywords: amphotericin B, micelle, Candida albicans, safety evaluation, buccal tablet, antifungal effect

\section{Introduction}

Candida albicans is one of the major human fungal pathogens, which usually causes oral mucosal infections. ${ }^{1}$ Such infections are difficult to eradicate and may emerge as lethal depending on the immune status of patients. Particularly, patients with immunecompromised diseases such as AIDS and cancer are life-threatened by the infection of $C$. albicans. ${ }^{2,3}$ In these patients, $C$. albicans invade their oral cavities and make them even more painful. In general, Candida infections begin with the adherence and colonization of yeast cells on a biotic host surface to form microbial communities, the so-called "biofilms". Biofilms are heterogeneous communities of microorganisms 
entrapped in an extracellular matrix that limits the penetration of antimicrobial drugs and antibodies. ${ }^{4}$ The formation of $C$. albicans biofilms is a crucial virulence factor that allows $C$. albicans 30-2,000 times more resistant to several antifungal agents compared with their planktonic (or freeliving) counterparts..$^{5}$ Therefore, the development of novel approaches to inactivate $C$. albicans biofilms has a great clinical significance in treating $C$. albicans infections.

Amphotericin B (AmB) is considered to be the most effective antibiotic in the treatment of chronic fungal infections, even effective against azole-resistant fungus. However, the clinical use of this antifungal drug is limited by side effects and poor bioavailability deriving from its inherent low solubility in water $(<0.001 \mathrm{mg} / \mathrm{mL}) .{ }^{6}$ Reported adverse effects of AmB also mainly result from its low solubility, and include renal failure, hypokalemia, hypomagnesemia, and polyuria. ${ }^{7}$ Thus, much effort has been expended to increase the water solubility of AmB. ${ }^{6,8-10}$ Fungizone $^{\circledR}$ (Bristol-Myers Squibb, Rueil-Malmaison, France) has been launched first as a colloidal dispersion combined with sodium deoxycholate (DOC) to develop a micellar dispersion for encapsulating AmB, which improves the solubility of AmB. However, the infusion toxicity and nephrotoxicity, which may ascribe to the dimeric form obtained with DOC, using a technological process with $\mathrm{pH}$ changes, still frequently cause troublesome complications. ${ }^{11-13}$ Commercial products, including a lipid complex (Abelcet ${ }^{\circledR}$, Sigma-Tau, Gaithersburg, MD, USA) and a liposomal product (Ambisome ${ }^{\circledR}$, Astellas, Tokyo, Japan) that encapsulate AmB with hydrogenated soybean lecithin, phosphatidylcholine, and cholesterol (polyaggregate forms), have already come to the market. The toxicity incidence of these formulations becomes lower, and they are accepted well when administered in higher doses. ${ }^{14,15}$ Nevertheless, their widespread use has been limited on account of high costs and high-dose requirements. Thus, extensive research has been done to develop other cost-efficient delivery systems (such as micelles, nanospheres, and conjugates) with lower toxicity.

Micelles show great superiority in aspects of solubilizing, permeability, and lower toxicity. Amphiphilic block copolymers have been in use as pharmaceutical excipients in drug delivery for a long time, and their application is developing rapidly in pharmaceutics and nanomedicine. ${ }^{16}$ These amphiphilic polymeric molecules can spontaneously form core-shell structures as micelles in an aqueous medium. The hydrophobic cores serve as reservoirs for poorly water-soluble drugs. Encapsulation of hydrophobic drugs into micelles contributed to substantially increasing a drug's solubility/dispersibility in aqueous media. ${ }^{17}$ Previously, we have synthesized monomethoxy poly(ethylene glycol)-poly(epsilon-caprolactone) (MPEGPCL) amphiphilic block copolymer and verified that the solubility of some poorly water-soluble drugs could be enhanced by encapsulating into MPEG-PCL copolymer. ${ }^{18}$ Wiegand et al ${ }^{19-21}$ have suggested that cationic homopolymers polyethylenimine (PEI) possessed a significant inhibition to C. albicans and even influenced the growth of C. albicans biofilms. ${ }^{22}$ However, an increasing degree of branching and molecular weight of PEI is confirmed to raise its cytotoxicity both in vitro and in vivo. In order to facilitate the antifungal efficiency and decrease toxicity, low-molecular-weight (800 or 1,800 Da) PEIs can be connected with other groups through short biodegradable linkages. ${ }^{23}$ Therefore, MPEG-PCL could be linked with PEI forming biodegradable triblock copolymer as a new drug delivery system. In this research, PEI with molecular weight 1,800 $\mathrm{Da}$ is utilized, and the connection with MPEG-PCL lowers the toxicity of PEI as well.

Buccal tablet is a mucoadhesive system that represents a good recent alternative. The mucoadhesive buccal drug delivery system offers several advantages as compared with traditional methods of systemic drug administration. The tablets are designed to release the drug either unidirectionally by targeting buccal mucosa or multidirectionally into the saliva. ${ }^{24,25}$ In addition to this, the drug can be easily applied and localized to the application site and can be removed directly by patients from there if necessary. ${ }^{26,27} \mathrm{We}$ prepared buccal tablet as a controlled and effective dosage form to let the drug release to oral saliva. The bioadhesive polymers were employed to increase the residence time of the drug in the oral cavity and the localization of the drug in a particular region.

Hence, in this work, we aimed to prepare AmB/ monomethoxy poly(ethylene glycol)-poly(epsiloncaprolactone)-graft-polyethylenimine (MPP) micelles to improve the solubility, safety, and antifungal effect of $\mathrm{AmB}$ in the oral buccal application. The characteristics of micelles and buccal tablets were investigated. Safety and gastrointestinal toxicity were also explored. Furthermore, we examined the antifungal activity of AmB/MPP micelles against planktonic and biofilm state of $C$. albicans.

\section{Materials and methods Materials}

AmB (98\% purity) was purchased from Shanghai Ruichu Biological Technology Co., Ltd., Shanghai, China. Milk protein concentrate (MPC85) was from Jinan Heluo Trade Company Ltd., Jinan, China. Hydroxypropyl methylcellulose K4M, corn starch, anhydrous lactose, and magnesium stearate were all obtained from Xi'an Yue Lai Medical Technology Co., 
Ltd., Shaanxi, China. Methanol was obtained from Tianjin Tianli Chemical Reagent Co., Ltd., Tianjin, China. MPEGPCL diblock copolymer with a designed molecular weight of $2 \mathrm{k}-2 \mathrm{k}$ was obtained from our laboratory. All other chemicals used were of analytical or high-performance liquid chromatography (HPLC) grade. C. albicans strains (ATCC 76485) were kindly provided by the Department of Microbiology and Immunology, Xi'an Jiaotong University.

\section{Synthesis of MPEG-PCL-g-PEI copolymer}

The diblock copolymer MPEG-PCL was synthesized previously by our laboratory. ${ }^{18}$ After dissolving the copolymer, a few drops of triethylamine and an excess amount of acryloyl chloride were added dropwise to the MPEG-PCL anhydrous dichloromethane solution. The mixture was stirred for $8 \mathrm{~h}$ at $45^{\circ} \mathrm{C}$ under the protection of nitrogen, then the copolymer MPEG-PCL containing $\mathrm{C}=\mathrm{C}$ was collected. Subsequently, the earlier copolymer was dissolved in chloroform and a certain amount of PEI chloroform solution was added dropwise. The mixture was stirred for another $48 \mathrm{~h}$ at $45^{\circ} \mathrm{C}$ under nitrogen atmosphere. The products were obtained by cooled petroleum ether co-precipitation and purified through dialysis. The chemical composition was confirmed by ${ }^{1} \mathrm{H}-\mathrm{NMR}$ spectra.

\section{Preparation of AmB/MPP micelles}

AmB/MPP micelles were prepared by the thin-film dispersion method. ${ }^{28-30}$ First, $10 \mathrm{mg}$ of AmB and $100 \mathrm{mg}$ of MPP triblock copolymer were co-dissolved in $200 \mathrm{~mL}$ methanol with the help of ultrasound. The methanol was recovered by evaporating at $55^{\circ} \mathrm{C}$ under reduced pressure (RE-52A; Shanghai Ya Rong Biochemistry Instrument Factory, Shanghai, China) to form a uniform thin film. To obtain the solution of drug-loaded micelles, AmB/MPP film was rehydrated in $100 \mathrm{~mL}$ of deionized water at $50^{\circ} \mathrm{C}$ with gentle agitation. Afterward, the solution was filtered using a $0.22 \mu \mathrm{m}$ filter, and the filtrate was lyophilized as the sample for further characterization research.

\section{Characterizations of micelles}

\section{Size and zeta potential}

Particle size, size distribution, and zeta potential values were measured by Malvern Zetasizer Nano-ZS (Malvern Instruments Ltd., Malvern, UK) at $25^{\circ} \mathrm{C}$. Measurements were performed in triplicate for every prepared sample.

\section{Transmission electron microscopy (TEM)}

The morphological feature of AmB/MPP micelles was observed by transmission electron microscope (H-600;
Hitachi, Tokyo, Japan). Prior to examination, one drop of micelles suspension was loaded on a copper grid and left to adhere for $2 \mathrm{~min}$. The excess suspension was sucked with a filter paper and air-dried before observation.

\section{Drug loading (DL\%) and entrapment efficiency (EE\%)}

For quantitative assay, AmB/MPP micelles were broken up by dimethylformamide (DMF) with the help of sonication and analyzed by HPLC. ${ }^{31-33}$ DL\% and EE\% were calculated as follows: ${ }^{34,35}$

$$
\begin{gathered}
\mathrm{DL} \%=\frac{\text { Amount of drug in micelles }}{\text { Amount of polymer }+ \text { drug }} \times 100 \% \\
\mathrm{EE} \%=\frac{\text { Amount of drug in micelles }}{\text { Amount of adding drug initially }} \times 100 \%
\end{gathered}
$$

\section{Differential scanning calorimetry (DSC)}

The formation of the inclusion complex between $\mathrm{AmB}$ and MPP was confirmed by DSC (DSC822 $2^{\mathrm{e}}$, Mettler Toledo, Greifensee, Switzerland). Each sample weighing $3 \mathrm{mg}$ was heated in hermetically sealed aluminum pans at a rate of $10^{\circ} \mathrm{C} / \mathrm{min}$ up to $250^{\circ} \mathrm{C}$.

\section{In vitro release}

The release profile of AmB was analyzed in phosphate-buffered saline (PBS; pH 5.8 and 6.8, each containing Tween-80, $0.5: 100, \mathrm{w} / \mathrm{v})$. A total of $1 \mathrm{~mL}$ of each sample was placed in a dialysis bag (molecular weight cut off $3.5 \mathrm{KDa}$ ). The dialysis bag was inserted in $20 \mathrm{~mL}$ of PBS with gentle shaking $(100 \mathrm{rpm})$ in a water bath at $37^{\circ} \mathrm{C}$. At specific time intervals, $0.5 \mathrm{~mL}$ of each sample was withdrawn from the external aqueous solution, and an equal amount of the dissolution medium was added in order to maintain sink conditions. Subsequently, the concentration of $\mathrm{AmB}$ released at various times up to $8 \mathrm{~h}$ was determined by HPLC. This analysis was performed on a Dikma C18 column $(250 \times 4.6 \mathrm{~mm}, 5 \mu \mathrm{m})$ with the injection volume of $20 \mu \mathrm{L}$, with acetonitrile: $10 \mathrm{mmol}$ sodium acetate aqueous solution (40:60) as mobile phase at a flow rate of $1 \mathrm{~mL} / \mathrm{min}$. The detector was at the wavelength of $408 \mathrm{~nm}$, and the column temperature was set at $30^{\circ} \mathrm{C}$. All release experiments were repeated three times.

\section{Preparation of buccal tablets with AmB/MPP micelles}

Prior to production, micelle solution was lyophilized to obtain freeze-dried powder, which can be compressed with other excipients. The freeze-dried powder and excipients without lubricant were blended in the order of ascending 
weights with gentle shaking. Then magnesium stearate was added and mixed again. Buccal tablets were prepared by direct compression process using a single punch tablet press (DP-30; Beijing Gylongli Sci. \& Tech. Co., Ltd., Beijing, China) at 1 ton pressure. Three formulations were made including AmB+DOC physical mixtures (T1), AmB+MPP physical mixtures (T2), and AmB/MPP micelles (T3). Details of the compositions are listed in Table 1. Evaluation of buccal tablets is given in the Supplementary materials.

\section{In vitro release of buccal tablets}

\section{Using the United States Pharmacopeia (USP)} dissolution apparatus

In vitro release characteristics of the mucoadhesive buccal tablets were investigated by basket stirring method using the USP dissolution test apparatus, and the accumulative release percentage was calculated. A total of $200 \mathrm{~mL}$ of sodium dodecyl sulfate (SDS) aqueous solution $(0.5 \%, \mathrm{w} / \mathrm{v})$ was selected as the release medium at $37^{\circ} \mathrm{C}$ and shaken at $50 \mathrm{rpm}$. At predetermined periods, $2.5 \mathrm{~mL}$ of the release medium was withdrawn and an equal volume of fresh medium $\left(37^{\circ} \mathrm{C}\right)$ was added back to maintain sink condition. The samples were filtered and analyzed by HPLC. All release experiments were repeated three times.

\section{Using the dissolution rate test apparatus}

To evaluate drug release in vivo, the dissolution rate test apparatus (China Patent no ZL201320445613.7) was designed for simulating in vivo conditions of the oral cavity. ${ }^{18}$ Each tablet was adhered to the cap of the dissolution cell using $20 \mu \mathrm{L}$ of artificial saliva $\left(500 \mathrm{~mL}\right.$ containing $5 \mathrm{mmol} / \mathrm{dm}^{3}$ sodium bicarbonate, $7.36 \mathrm{mmol} / \mathrm{dm}^{3}$ sodium chloride, $20 \mathrm{mmol} / \mathrm{dm}^{3}$ potassium chloride, $6.6 \mathrm{mmol} / \mathrm{dm}^{3}$ sodium dihydrogen phosphate monohydrate, and $1.5 \mathrm{mmol} / \mathrm{dm}^{3}$ calcium chloride

Table I Composition of different buccal tablets

\begin{tabular}{llll}
\hline Composition & \multicolumn{3}{l}{ Content (mg) } \\
\cline { 2 - 4 } & TI & T2 & T3 \\
\hline MPC85 & 27.43 & 27.43 & 27.43 \\
HPMC K4M & 20.50 & 20.50 & 20.50 \\
Corn starch & 58.36 & 49.18 & 49.18 \\
Anhydrous lactose & 5.57 & 5.57 & 5.57 \\
Magnesium stearate & 1.32 & 1.32 & 1.32 \\
AmB & 1.00 & 1.00 & - \\
MPEG-PCL-g-PEI & - & 10.00 & - \\
AmB/MPEG-PCL-g-PEI & - & - & 11.00 \\
DOC & 0.82 & - & - \\
\hline
\end{tabular}

Note: TI, AmB+DOC; T2, AmB+MPP; T3, AmB/MPP micelles.

Abbreviations: AmB, amphotericin B; MPEG-PCL-g-PEl, monomethoxy poly (ethylene glycol)-poly(epsilon-caprolactone)-graft-polyethylenimine; DOC, sodium deoxycholate; MPP, monomethoxy poly(ethylene glycol)-poly(epsilon-caprolactone)graft-polyethylenimine. dehydrate, adjusted to $\mathrm{pH} 6.8$ or 5.8) and lightly pressed for $30 \mathrm{~s} .{ }^{36}$ After assembling the cap to the dissolution cell, artificial saliva was pumped through the cell with a peristaltic pump to control the velocity at $\sim 0.6 \mathrm{~mL} / \mathrm{min} .{ }^{26,37}$ A total of $1.5 \mathrm{~mL}$ continuous flow of artificial saliva suffused the dissolution cell and was agitated with a magnetic stirrer at $50 \mathrm{rpm}$. The apparatus was maintained at $37^{\circ} \mathrm{C}$ in a thermostat bath. Every fractional sample was collected at predetermined periods and analyzed by HPLC. Six tablets of each formulation were analysed. The accumulative release amount $\left(Q_{\mathrm{n}}\right)$ of the drug at different times was calculated as follows:

$$
Q_{n}(\%)=\frac{\left[C_{n} \times V_{n}+\sum_{i=1}^{n-1} C_{i} \times V_{i}\right]}{W} \times 100 \%
$$

where $C_{n}$ indicated the drug concentration of sample $n$ at determined times; $V_{n}$ showed the volume of the collected artificial saliva; and $W$ was the labeled amount (mg) of the buccal tablet.

\section{Cytotoxicity assay of the micelles}

To evaluate the cytotoxicity of AmB/MPP micelles by MTT assay, KB cells were inoculated at a density of $1 \times 10^{4}$ cells/well in a 96-well plate overnight. Then, the culture medium was removed by pipette. Fungizone (a micellar dispersion of AmB with DOC), ${ }^{38,39}$ AmB/MPP micelles, and MPP blank micelles were added to each well and incubated at $37^{\circ} \mathrm{C}$ for a certain time. Following incubation, $20 \mu \mathrm{L}$ of $5 \mathrm{mg} /$ $\mathrm{mL}$ MTT in PBS was put into each well and then incubated for $4 \mathrm{~h}$ at $37^{\circ} \mathrm{C}$. To dissolve the formazan crystal, $150 \mu \mathrm{L}$ of dimethyl sulfoxide (DMSO) was added. After shaking the 96-well plate for $30 \mathrm{~s}$, the absorbance was measured at $492 \mathrm{~nm}$ by a microplate reader (BioTek Epoch, Winooski, VT, USA). The $100 \%$ value as the standard to assess cytotoxicity was obtained from the optical density (OD) values assayed in untreated cells.

\section{Histopathology of oral buccal mucosal tissues}

Male Sprague Dawley rats (230 $\pm 10 \mathrm{~g})$ were obtained from the Lab Animal Center of Xi' an Jiaotong University (Shaanxi, China). The experimental protocol was in accordance with the National Institutes of Health Guide for Care and Use of Laboratory Animals and was approved by the Institutional Animal Care Committee of Xi'an Jiaotong University. Before the experiments, rats were raised in an environmentally controlled breeding room for 1 week and fed with standard laboratory food and water. The control group was 
the $5 \%$ dextrose sterile solution, and other groups (including Fungizone, AmB/MPP micelles, and MPP blank micelles) were dissolved in the $5 \%$ dextrose sterile solution. Then, $50 \mu \mathrm{L}$ of each formulation was injected into the oral buccal mucosal tissues of the rats. At the end of treatments (after injection for 5,15 , and 30 days), some rats were sacrificed by injecting air into the thoracic cavity. Oral buccal mucosal tissues were isolated and fixed in 10\% neutral carbonatedbuffered formaldehyde. The fixed tissues were optimal cutting temperature-embedded and sliced using a freezing microtome. Hematoxylin and eosin-stained sections of the oral buccal mucosal tissues were evaluated.

\section{Ultrastructure observation of the tissues}

Before the observation, the isolated oral buccal mucosal tissues were cut into small pieces of size $0.5-1 \mathrm{~mm}^{3}$ and directly placed into the precooling glutaraldehyde solution at $4^{\circ} \mathrm{C}$. At least $4 \mathrm{~h}$ later, samples were rinsed with $0.1 \mathrm{~mol} / \mathrm{L} \mathrm{PBS}$ three times and then fixed with 1\% osmic acid. Epon 812 was the embedding medium. Ultrastructure observation of the oral buccal mucosal tissues was analyzed under transmission electron microscope (H-7650; Hitachi).

\section{Intragastric administration of the micelles}

For toxicity analysis of the micelles through the gastrointestinal tract, AmB/MPP micelles in the 5\% dextrose sterile solution were intragastrically administrated through the stomach sondle needle at the dose of $5 \mathrm{~mL} / \mathrm{kg}$. Then, blood samples $(0.2 \mathrm{~mL})$ were withdrawn in heparinized tubes from the femoral artery using cannulation at $0,15,30,60$, 120, 240, and $480 \mathrm{~min}$ after oral administration. The blood samples were centrifuged at $3,000 \times g$ for $5 \mathrm{~min}$, and the plasma samples were harvested and stored at $-20^{\circ} \mathrm{C}$ until liquid chromatography-mass spectrometry (LC-MS) analysis (LC-MS method is given in the Supplementary materials).

\section{Antifungal activity}

The minimal inhibitory concentration (MIC) for planktonic $C$. albicans was determined using the microdilution method according to the guidelines of the M27-A3 document of the Clinical and Laboratory Standards Institute. The fungal cells were seeded on a 96-well microtiter plate in Roswell Park Memorial Institute 1640 medium at a density of $1 \times 10^{5}$ cells $(100 \mu \mathrm{L}$ per well $)$, and the final drug concentrations were $0.098-50 \mu \mathrm{g} / \mathrm{mL}$ for AmB/MPP micelles and $\mathrm{AmB}$. Plates were incubated at $30^{\circ} \mathrm{C}$ for 48 h. Then, 2,3-bis(2-methoxy-4-nitro-5-sulfophenyl)2H-tetrazolium-5-carboxanilide (XTT)-menadione solution was added and finally measured at the absorbance of $490 \mathrm{~nm}$ using a microplate reader (BioTek Epoch). Experiments were performed three times with six replicate wells for each experiment.

\section{Formation and quantification of biofilms}

Biofilms were prepared in flat-bottomed 96-well microplates adapted from a method of O'Toole and Kolter, ${ }^{40}$ which was based on the ability of microorganisms to form biofilms on solid surfaces and used crystal violet (CV) to stain biofilms. Briefly, $100 \mu \mathrm{L}$ of $1 \times 10^{5}$ cells $/ \mathrm{mL}$ suspension in Roswell Park Memorial Institute 1640 medium was inoculated at $37^{\circ} \mathrm{C}$ without shaking for $48 \mathrm{~h}$, forming the biofilms. The morphology of biofilms was observed under scanning electron microscope (SEM; TH-1000; Hitachi). Biofilms were washed with sterile $\mathrm{PBS}$ and incubated at $37^{\circ} \mathrm{C}$ for $48 \mathrm{~h}$ with different concentrations of drugs. Plates were gently rinsed with PBS and air-dried for $24 \mathrm{~h}$ prior to $\mathrm{CV}$ staining. Adherent films of each well were stained with $200 \mu \mathrm{L}$ per well of $1 \%(\mathrm{w} / \mathrm{v})$ $\mathrm{CV}$ (5 min) and then washed with sterile PBS to remove unbound dye. Afterward, CV was extracted with $200 \mu \mathrm{L}$ of the bleaching solution: ethanol/glacial acetone (70:30), and biofilms were quantified by measuring the OD at $595 \mathrm{~nm}$ with the microplate reader. The biofilm biomass unit (BBU) was arbitrarily defined with $0.1 \mathrm{OD}_{595}$ equal to $1 \mathrm{BBU}$.

\section{Statistical analysis}

The statistical analyses were performed using Student's $t$-test with a level of significance at $P<0.05$.

\section{Results and discussion Preparation and characterization of micelles}

The copolymer MPEG-PCL-g-PEI was synthesized, and the chemical composition was confirmed by ${ }^{1} \mathrm{H}-\mathrm{NMR}$ spectra (Figure 1B). In the aqueous solution, this triblock copolymer could self-assemble into nanoscale micelles when heated to $50^{\circ} \mathrm{C}$ (Figure 1A). Poorly water-soluble AmB was encapsulated into the inner hydrophobic core of the copolymer on account of the hydrophobic interaction. The encapsulation efficiency and drug loading were $94.11 \% \pm 1.25 \%$ and $8.60 \% \pm 0.21 \%$, respectively.

The average size of blank micelles and drug-loaded micelles (Figure 2A) was $149.7 \pm 0.7 \mathrm{~nm}$ (polydispersity index $[\mathrm{PDI}]=0.297 \pm 0.017)$ and $148.1 \pm 0.9 \mathrm{~nm}(\mathrm{PDI}=0.279 \pm 0.018)$, respectively. These size distribution spectrums suggested that there was no significant variation in the particle size between the drug-loaded micelles and blank micelles. As shown in Figure 2B, zeta potential of blank micelles and drug-loaded micelles was $\sim 25.5 \pm 1.67$ and $17.2 \pm 0.62 \mathrm{mV}$, 
A

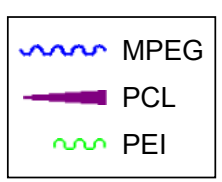

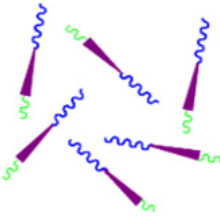

MPEG-PCL-g-PEI
Amphotericin B

B
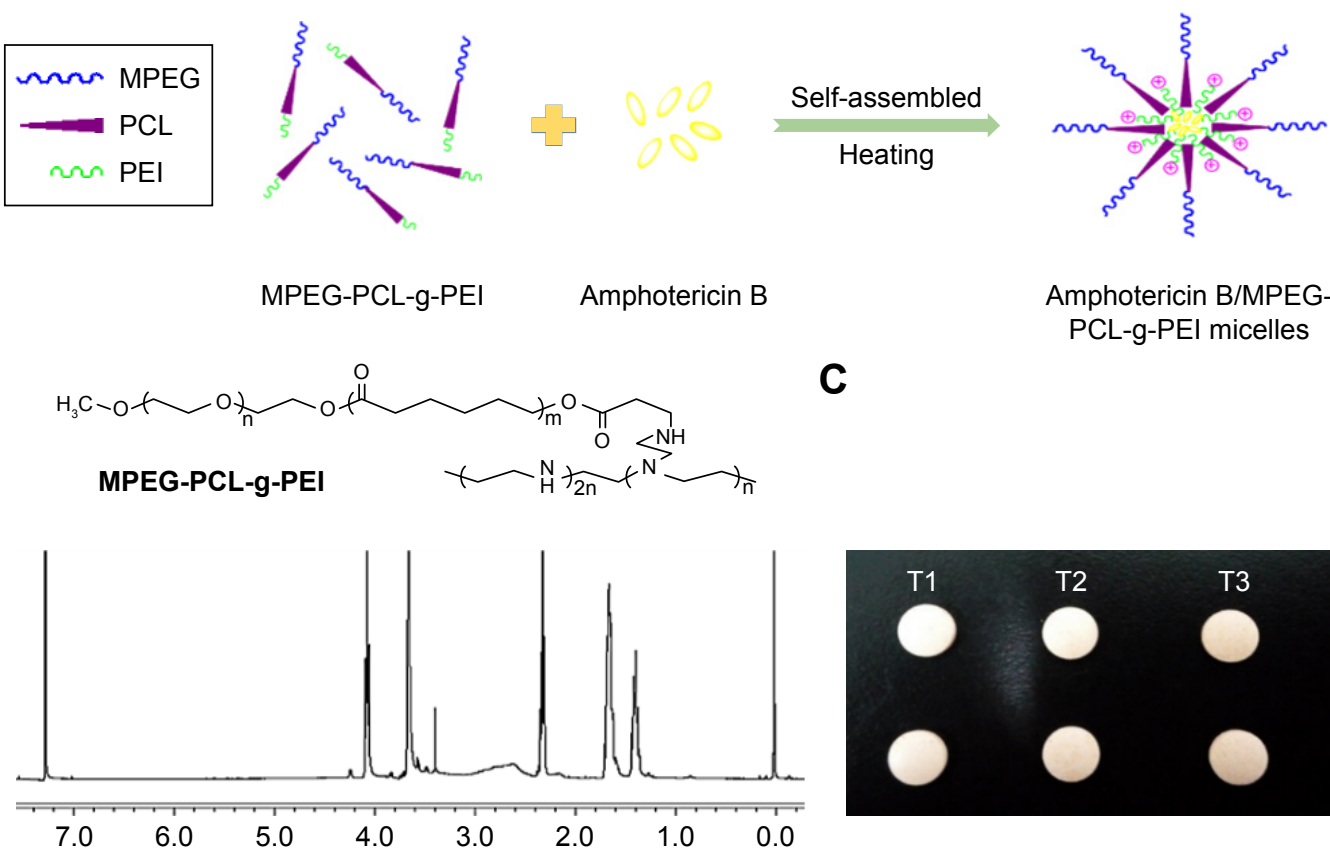

Amphotericin B/MPEG-

PCL-g-PEI micelles

\section{Self-assembled \\ Heating}

C

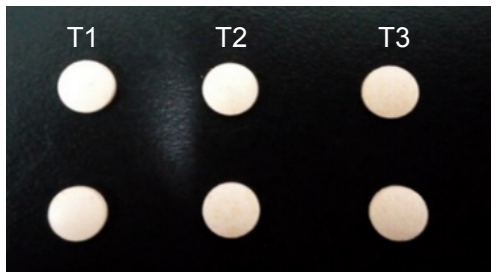

Figure I (A) Spontaneous AmB-loaded micelles formation from amphiphilic molecules in aqueous media; (B) the chemical formula and the 'H-NMR spectra (400 MHz, $\mathrm{CDCl}_{3}$ ) of MPEG-PCL-g-PEl; (C) buccal tablets containing AmB+DOC (TI), AmB+MPEG-PCL-g-PEl physical mixtures (T2), and AmB/MPEG-PCL-g-PEI micelles (T3).

Abbreviations: AmB, amphotericin B; MPEG-PCL-g-PEI, monomethoxy poly(ethylene glycol)-poly(epsilon-caprolactone)-graft-polyethylenimine; DOC, sodium deoxycholate.

respectively. These micelles showed positive charge, which indicated the linkage of cationic polymer PEI. The reduction of zeta potential may be related to the encapsulation of AmB, which was identical to that of the previous studies. ${ }^{41}$
Although zeta potential of drug-loaded micelles was lower than that of blank micelles, they all presented good dispersion and stability during the period of the experiment. Figure $2 \mathrm{C}$ exhibits the morphological observation of $\mathrm{AmB} /$
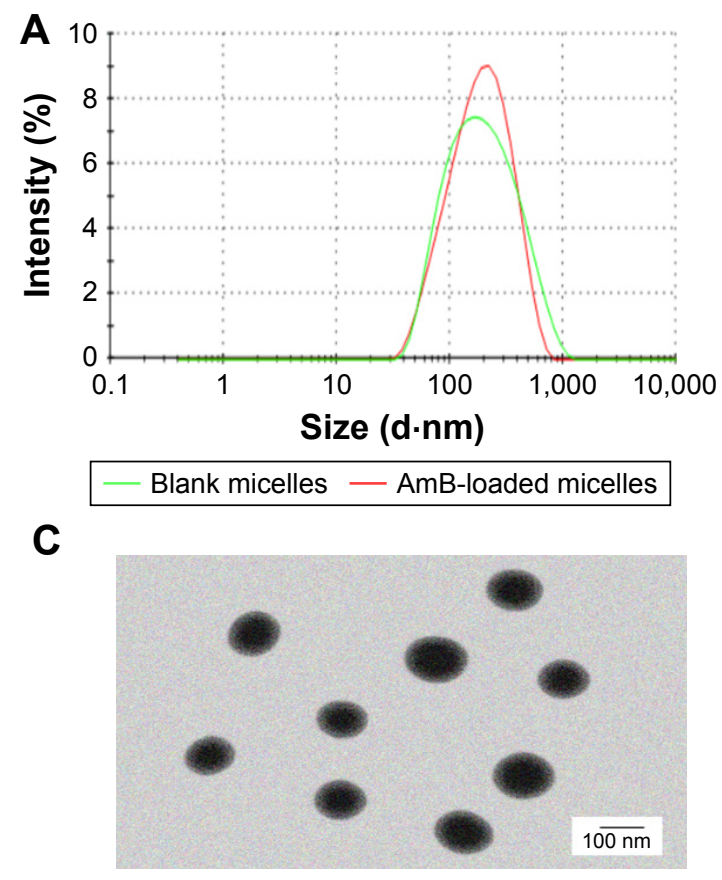

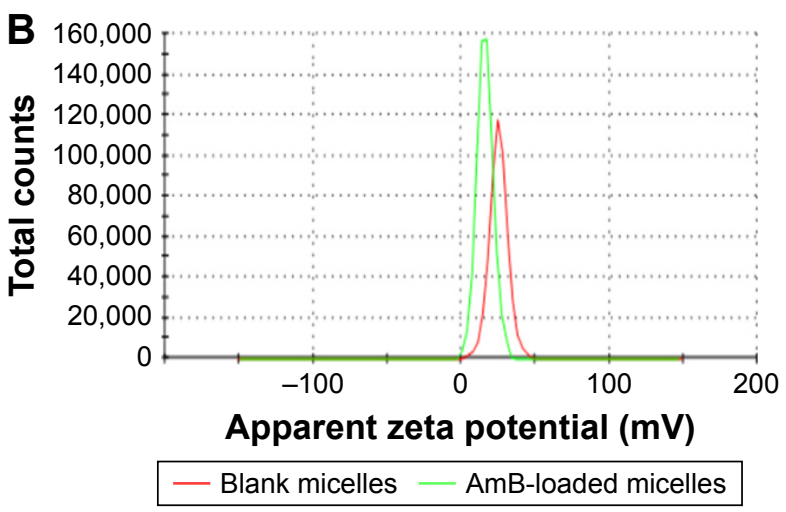

D

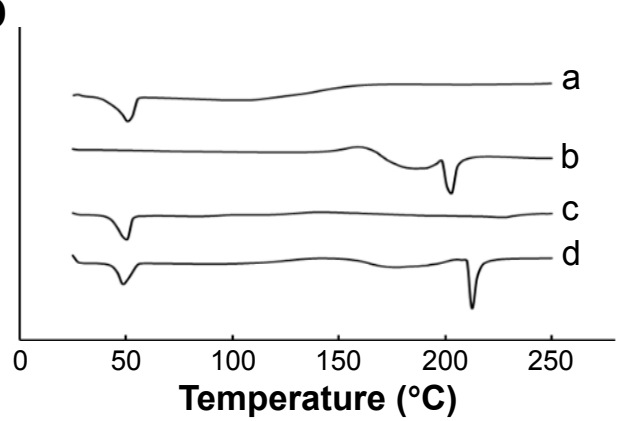

Figure 2 (A) The particle size distribution spectrum of blank micelles and AmB-loaded micelles; (B) zeta potential of blank micelles and AmB-loaded micelles; (C) morphology of AmB-loaded micelles determined by TEM (scale bar $100 \mathrm{~nm}$ ); (D) DSC analysis of (a) MPP, (b) AmB, (c) AmB/MPP micelles, and (d) AmB and MPP physical mixtures. Abbreviations: AmB, amphotericin B; TEM, transmission electron microscopy; DSC, differential scanning calorimetry; MPP, monomethoxy poly(ethylene glycol)poly(epsilon-caprolactone)-graft-polyethylenimine. 
MPP micelles. Most of the micelles appeared spherical in shape and dispersed uniformly. It could be inferred that the hydrophobic terminals of PCL chains formed the micellar core and the hydrophilic terminals of MPEG and PEI chains composed the hydrophilic corona.

DSC analysis is an effective identification method to validate the state of incorporated drugs. The DSC curves of AmB, MPEG-PCL-g-PEI, AmB and MPEG-PCL-g-PEI physical mixture, and AmB/MPP micelles are shown in Figure 2D. There was no evidence of melting in AmB up to $250^{\circ} \mathrm{C}$, because this antibiotic has already decomposed, which could be observed in the DSC thermogram for AmB (double broad endotherms at $\sim 170$ and $210^{\circ} \mathrm{C}$ ) and was identical to that of the previous report. ${ }^{42}$ MPEG-PCL-g-PEI showed a broad endothermic peak at $\sim 47^{\circ} \mathrm{C}$, while the DSC scan of AmB/MPEG-PCL-g-PEI micelles did not show any signs of $\mathrm{AmB}$ endothermic peak, indicating the formation of an amorphous state and the molecular encapsulation of the drug inside the core-shell structure.

\section{In vitro release of micelles}

The $\mathrm{pH}$ value in the oral cavity for healthy humans was found to be $\sim 6.3-7.4,{ }^{43,44}$ while it decreased to weak acid condition for patients who suffered from oral C. albicans. In this research, in vitro release profile of AmB/MPP micelles and redissolved micelles (lyophilized powder redissolved in water) in different mediums ( $\mathrm{pH} 5.8$ and 6.8 and $0.5 \%$ SDS aqueous solution) was investigated (Figure 3). Mediums ( $\mathrm{pH} 5.8$ and 6.8 ) contained $0.5 \%$ Tween-80 (w/v). It could be observed that there was no significant difference between micelles and redissolved micelles release; thus, lyophilized

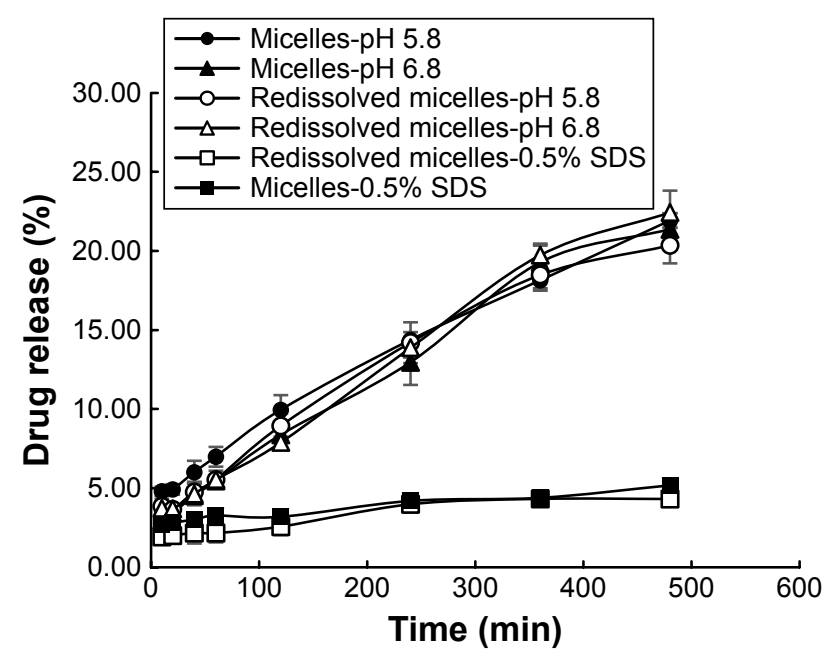

Figure 3 Release profile of AmB-loaded micelles and redissolved micelles in different mediums.

Abbreviations: AmB, amphotericin B; SDS, sodium dodecyl sulfate. powder redissolved in water was utilized in further research. In the $\mathrm{pH} 5.8$ and 6.8 buffer solutions, AmB release was dramatically faster than that in the $0.5 \%$ SDS aqueous solution ( $\mathrm{pH} 8.0$ ); probably the different ionic strength of the media could influence the release. It seems that $\mathrm{pH} 5.8$ and 6.8 buffer solutions were more suitable for the evaluation of the micelles release. Besides, no obvious difference was observed between $\mathrm{pH} 5.8$ and 6.8 buffer solutions for AmB release. As well known, AmB was insoluble in water, and its solubility could be enhanced when encapsulated into the MPEG-PCL-g-PEI micelles in both normal conditions (pH 6.8) and pathological conditions ( $\mathrm{pH}$ 5.8) without dependence on the $\mathrm{pH}$ changes.

\section{Evaluation of buccal tablets}

The buccal tablets were produced according to the formulation composition in Table 1, and the evaluation results are shown in Table 2. The weight variation, hardness, and friability parameters of the prepared buccal tablets were within the range of Chinese pharmacopoeial limit. The parameters of buccal tablets' weight, size, and hardness were selected on the basis of Oravig ${ }^{\circledR}$ (Miconazole buccal tablet, approved by the Food and Drug Administration [FDA] since 2010), which take into consideration patients' tolerance. ${ }^{45}$ The picture of the tablets is shown in Figure 1C.

\section{In vitro drug release studies of buccal tablets}

For USP dissolution apparatus release, as shown in Figure 4A, there was no burst release phenomenon in any AmB buccal tablet formulation. MPC85 and HPMC K4M in the prescription were employed as mucoadhesive polymers. A $0.5 \%$ SDS aqueous solution was used in the dissolution medium for miconazole buccal tablet in FDA dissolution methods. T1 was the control group mainly including AmB and DOC, which had the same ingredient with Fungizone. ${ }^{46,47}$ T2 consisted of the physical mixtures of AmB and MPP. AmB/ MPP micelles took up the major component of T3. T2 was designed to prove the solubilization capacity of the core-shell structure of the micelles compared with the physical mixtures.

Table 2 Evaluation results of buccal tablets

\begin{tabular}{llll}
\hline FC & Weight variation $^{\mathbf{a}}(\mathbf{m g})$ & Hardness $^{\mathbf{a}}(\mathbf{k g})$ & Friability $^{\mathbf{a}} \mathbf{( \% )}$ \\
\hline TI & $115.78 \pm 0.7 \mathrm{I}$ & $2.56 \pm 0.42$ & $0.78 \pm 0.14$ \\
T2 & $114.20 \pm 1.21$ & $2.26 \pm 0.27$ & $0.69 \pm 0.23$ \\
T3 & $116.32 \pm 0.98$ & $2.38 \pm 0.36$ & $0.65 \pm 0.12$
\end{tabular}

Notes: ${ }^{\text {Mean }} \pm \mathrm{SD}, \mathrm{n}=3$. TI, AmB+DOC; T2, AmB+MPP; T3, AmB/MPP micelles. Abbreviations: FC, formulation composition; SD, standard deviation; $A m B$, amphotericin B; DOC, sodium deoxycholate; MPP, monomethoxy poly(ethylene glycol)-poly(epsilon-caprolactone)-graft-polyethylenimine. 

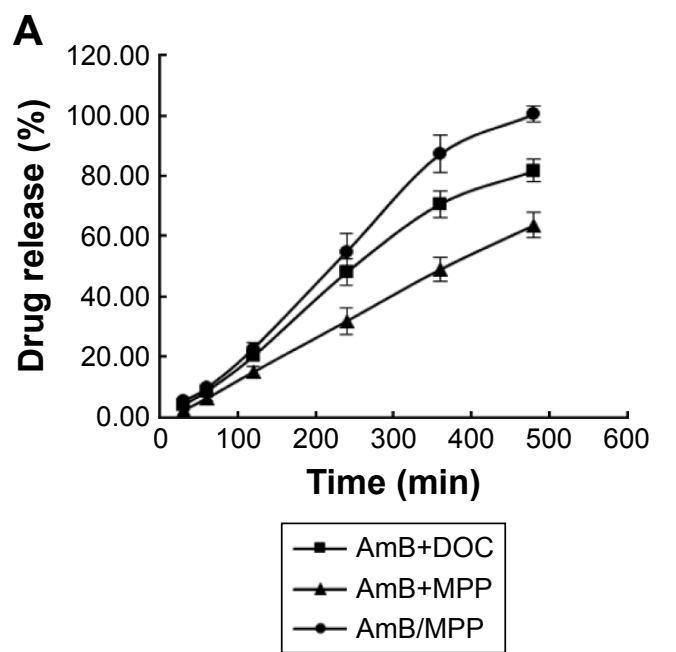

B

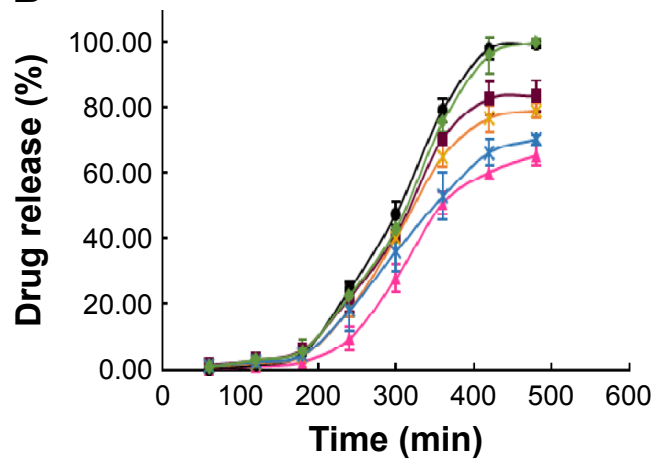

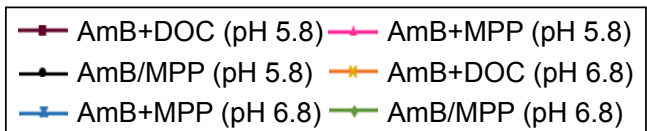

Figure 4 The release profiles of AmB from buccal tablets using different dissolution apparatuses.

Notes: (A) USP dissolution apparatus (in 0.5\% SDS solution) and (B) the dissolution rate test apparatus (in pH 5.8 and pH $6.8 \mathrm{medium}$ ). TI, AmB+DOC; T2, AmB+MPP; T3, AmB/MPP micelles.

Abbreviations: AmB, amphotericin B; SDS, sodium dodecyl sulfate; DOC, sodium deoxycholate; MPP, monomethoxy poly(ethylene glycol)-poly(epsilon-caprolactone)graft-polyethylenimine; USP, United States Pharmacopeia.

It was obvious that $\mathrm{AmB}$ was able to release $\sim 100 \%$ at $8 \mathrm{~h}$ for T3, and the cumulative release amount was significantly higher than T1. Thus, it could be inferred that this core-shell structure of the micelles has advantages on dissolution and improves the release of hydrophobic drugs. The buccal tablet T3 we prepared could meet the release requirements and exert medicinal effect effectively and continuously.

The in vitro release results of the buccal tablets were similar between our designed dissolution rate test apparatus (Figure 4B) and USP dissolution apparatus. Both the apparatuses could be utilized in the evaluation of in vitro release for the buccal tablets. All the results demonstrated that MPEG-PCL-g-PEI micelles could improve the solubility of AmB.

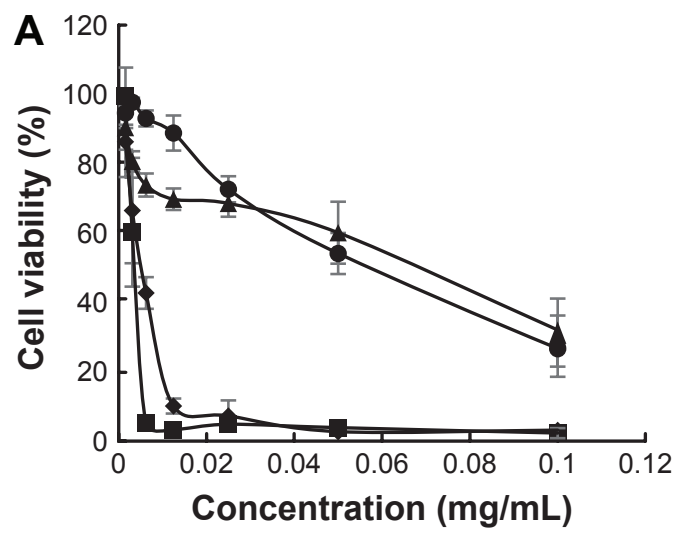

\section{Cytotoxicity assay of the micelles}

The cytotoxicities of AmB/MPP micelles were evaluated by MTT assay using KB cells (Figure 5A and B). Cell viabilities $(\%)$ of different concentrations of free AmB, AmB+DOC, AmB/MPP micelles, and MPP blank micelles were investigated. Although AmB reduced the viability of the cells in a dose-dependent manner, the use of MPP micelles significantly lowered the cytotoxicity of AmB. The cell viabilities of AmB/MPP micelles and MPP blank micelles were much higher than those of free $\mathrm{AmB}$ and $\mathrm{AmB}+\mathrm{DOC}$. It could be observed that the MTT results after dosing for 12 or $24 \mathrm{~h}$ had no obvious difference. Both these results proved that AmB/ MPP micelles and MPP blank micelles were less cytotoxic than free $\mathrm{AmB}$ and $\mathrm{AmB}+\mathrm{DOC}$.

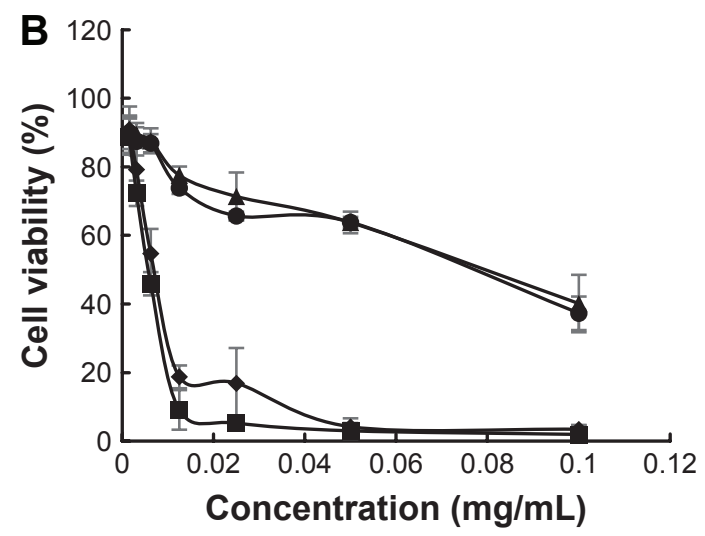

$$
\longrightarrow \text { AmB/MPP } \longrightarrow \text { MPP } \longrightarrow \text { Fungizone } \longrightarrow \text { AmB }
$$

Figure 5 Cytotoxicity of AmB, Fungizone (AmB+DOC), AmB/MPP micelles, and MPP blank micelles incubation for (A) $24 \mathrm{~h}$ and (B) $12 \mathrm{~h}$. Abbreviations: AmB, amphotericin B; DOC, sodium deoxycholate; MPP, monomethoxy poly(ethylene glycol)-poly(epsilon-caprolactone)-graft-polyethylenimine. 


\section{Histopathology of oral buccal mucosal tissues}

Safety is a critical issue driving the design or evaluation for a drug delivery system. Given the cell cytotoxicity results, we next examined the histology of the oral buccal mucosal tissues after administration. All the drugs were dissolved in $5 \%$ dextrose solution and filtered before use. Right buccal tissues of the rats were treated with $50 \mu \mathrm{L}$ of $\mathrm{AmB}+\mathrm{DOC}$, $\mathrm{AmB} / \mathrm{MPP}$, and MPP micelles through injection into the buccal mucosal tissues, and left buccal tissues were instilled with an equal volume of $5 \%$ dextrose solution as negative control groups. After administration over 5, 15, and 30 days, there were no discernible effects on the histopathology of oral mucosa for $5 \%$ dextrose solution, AmB/MPP, and MPP micelle groups (Figure 6). Histopathology data showed neither inflammation nor tissue necrosis after administration for 5 days. However, for AmB+DOC group, visible signs of break at the surface, middle, and base level of buccal epithelium membrane emerged from the oral mucosa. ${ }^{48}$ Until administration over 30 days, there was a slight recovery in the oral mucosa. As compared to the $5 \%$ dextrose solution group, AmB/MPP- and MPP-treated ones showed no visible sign of pathological changes.

\section{Ultrastructure observation of the tissues}

The histopathological examination of oral buccal mucosal tissues was done in order to evaluate the changes of the intermediate layer in mucosa epithelium and base layer cells; so ultrastructure observation of the tissues was also required. ${ }^{49-53} \mathrm{We}$ analysed the buccal tissues after dosing for 30 days with $5 \%$ dextrose solution, $\mathrm{AmB}+\mathrm{DOC}, \mathrm{AmB} /$ MPP, and MPP groups (Figure 7). In Figure 7A, the mucosal epithelial surface layer of the AmB+DOC group showed apparent swelling, so that only a partial surface layer could be observed in the same view compared with other groups. The epithelial intermediate layer (spinous layer) containing several prickle cells is shown in Figure 7B. For AmB+DOC group, the prickle cells were loose and the gap of intercellular space became bigger than other groups. Figure $7 \mathrm{C}$ shows the basement membrane of the mucosa; some parts in the basement membrane had disappeared in the AMB+DOC group, while other groups were intact. Basement membrane played
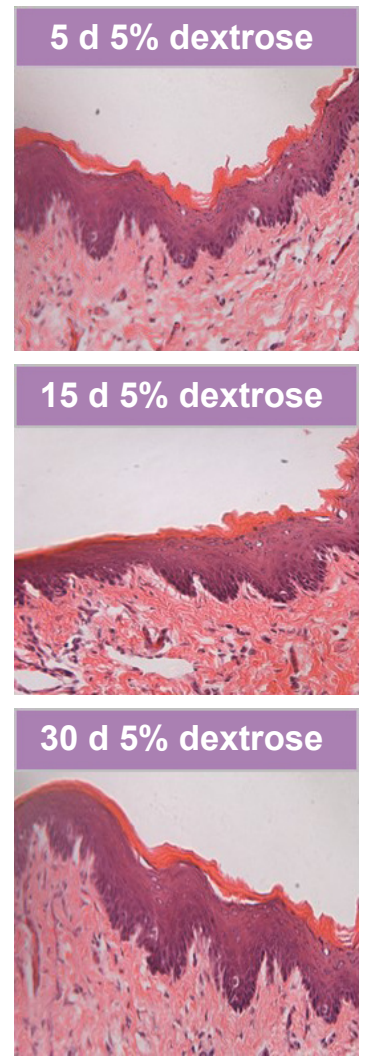
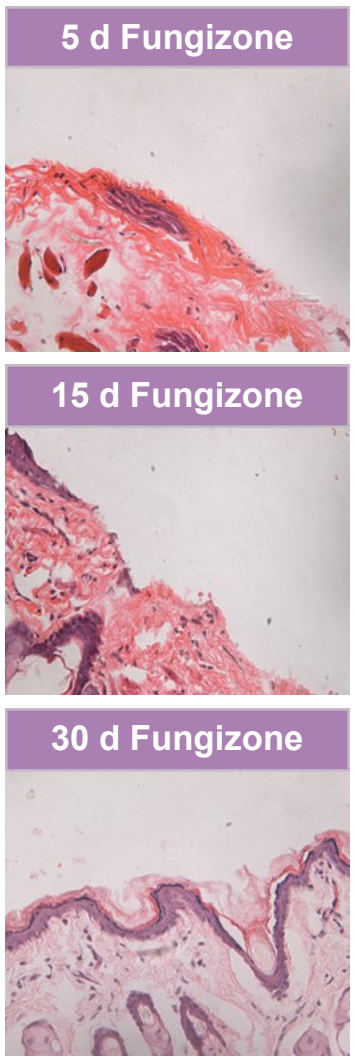
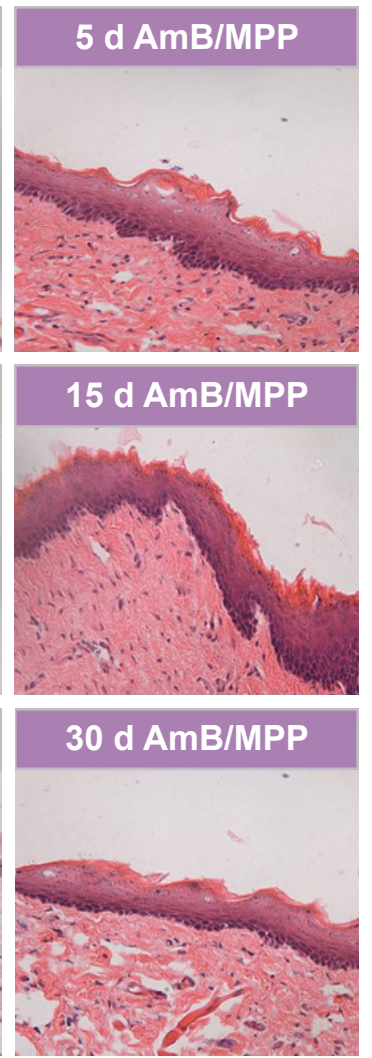
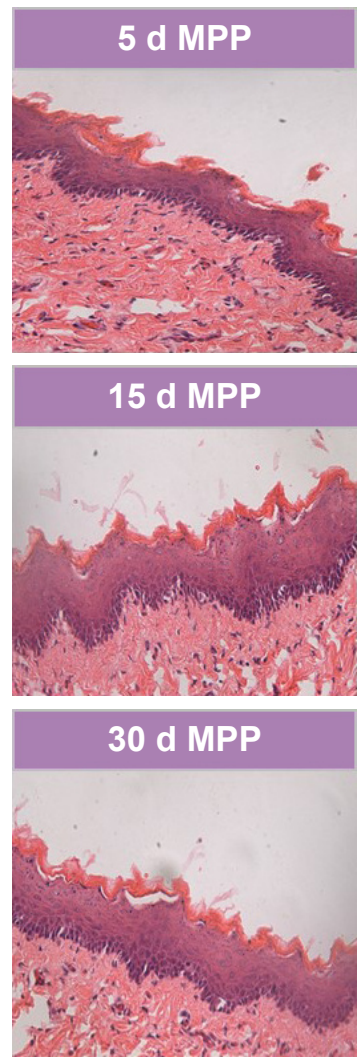

Figure 6 Histopathological observation of oral buccal tissues through injection with 5\% dextrose solution, Fungizone (AmB+DOC), AmB/MPP micelles, and MPP blank micelles under a light microscope (400x) after dosing for 5, 15, and 30 days.

Abbreviations: AmB, amphotericin B; DOC, sodium deoxycholate; MPP, monomethoxy poly(ethylene glycol)-poly(epsilon-caprolactone)-graft-polyethylenimine; d, days. 

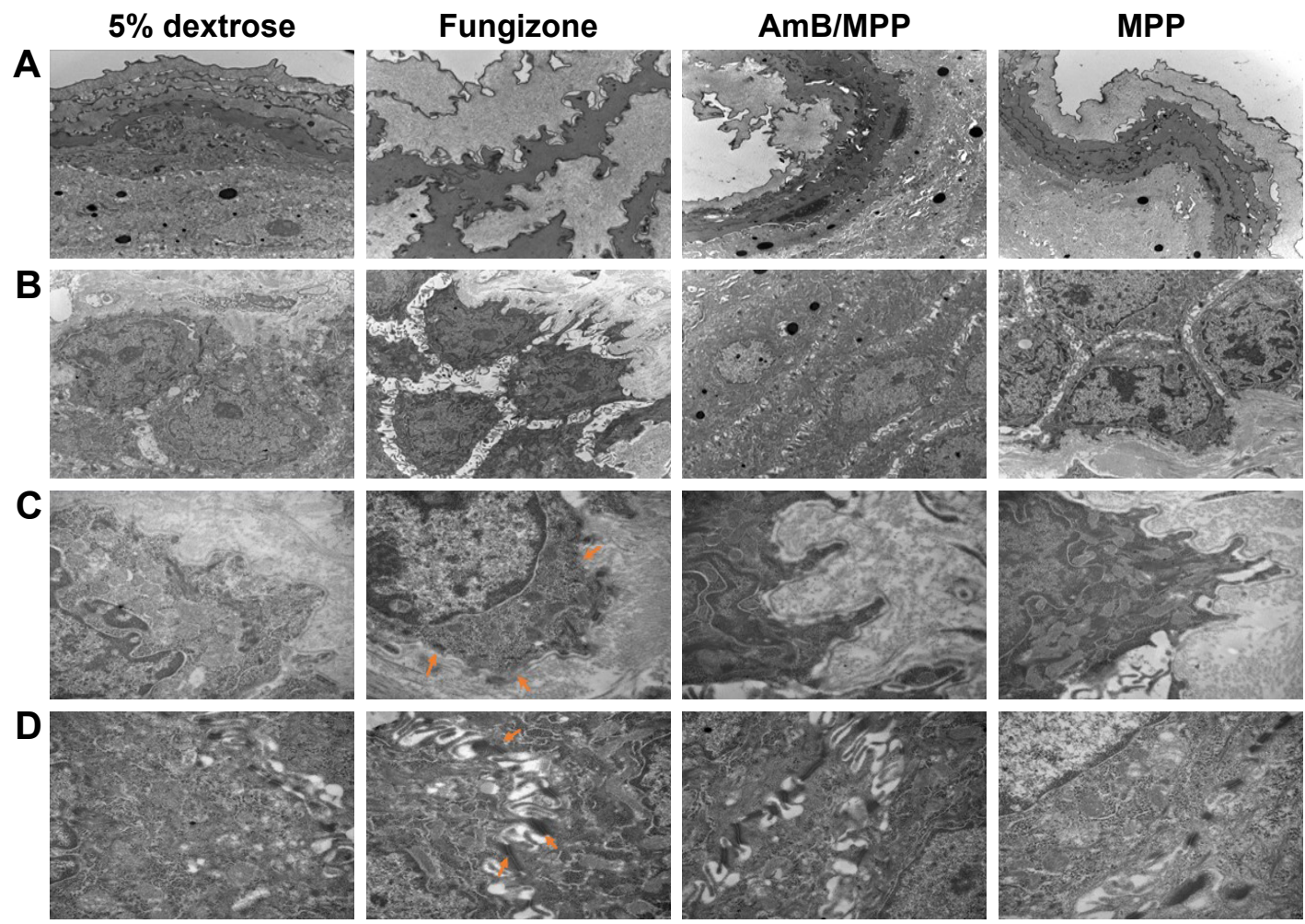

Figure 7 Ultrastructure observation of oral buccal tissues through injection with $5 \%$ dextrose solution, Fungizone (AmB+DOC), AmB/MPP micelles, and MPP blank micelles after dosing for 30 days under transmission electron microscope.

Notes: (A) Epithelial surface layer of the oral buccal tissues (I0,000x); (B) epithelial middle layer containing prickle cells (10,000x); (C) basement membrane (30,000x, orange arrows pointed to the disappeared parts in the basement membrane of the AMB+DOC group); (D) desmosome junctions between epithelial cells (30,000X, orange arrows pointed to the residual desmosome junctions in $A m B+D O C$ group).

Abbreviations: AmB, amphotericin B; DOC, sodium deoxycholate; MPP, monomethoxy poly(ethylene glycol)-poly(epsilon-caprolactone)-graft-polyethylenimine.

a major role on support, connection, and fix effect to the tissue. Besides, it was also a barrier structure for permeability, facilitating blood and material exchange between epithelial cells. Therefore, the basilar membrane had vital significance for the physiological functions of buccal mucosa tissue. In Figure 7D, desmosome junctions between epithelial cells were able to reflect the stability state of the tissue, namely, the more desmosome junctions the greater stability of the tissue. However, desmosome junctions in $\mathrm{AmB}+\mathrm{DOC}$ group were less than those in other groups. As compared to the 5\% dextrose solution group, the AmB/MPP- and MPP-treated ones exhibited less pathological changes. The inner components of prickle cells and basal cells in the $\mathrm{AmB}+\mathrm{DOC}$ group showed no obvious lesion, which may be attributed to the recovery of the animals after dosing.

\section{Gastrointestinal administration of the micelles}

When AmB/MPP micelles were employed in the buccal application, it seems possible that the micelles could be swallowed into the gastrointestinal environment. To analyze the toxicity of the micelles through the gastrointestinal tract, AmB/MPEG-PCL-g-PEI micelles were intragastrically administrated to the rats. In this experiment, the blood samples were withdrawn at $0,15,30,60,120,240$, and $480 \mathrm{~min}$ after oral administration. After LC-MS detection of the plasma samples, no AmB absorption was detected until $8 \mathrm{~h}$ (Supplementary materials). This verified that AmB/MPP micelles in the buccal application would not be ingested into the blood if unnecessary swallowing occurred. In consequence, AmB/MPP micelles in the oral cavity, local application, would not cause systemic toxicity, especially for nephrotoxicity.

\section{Antifungal effect of the micelles}

The biological studies showed that AmB/MPP micelles maintain high antimicrobial activity, which is similar to that of free AmB in DMSO (Figure 8A), whereas empty micelles had a significantly higher MIC value. This indicates that AmB-incorporated micelles have a similar antimicrobial potential to free AmB itself. In spite of the introduction of cation polymer, the antifungal effect to planktonic cells did not improve significantly. This phenomenon may be ascribed to the drug release process from the micelles, while the drug 

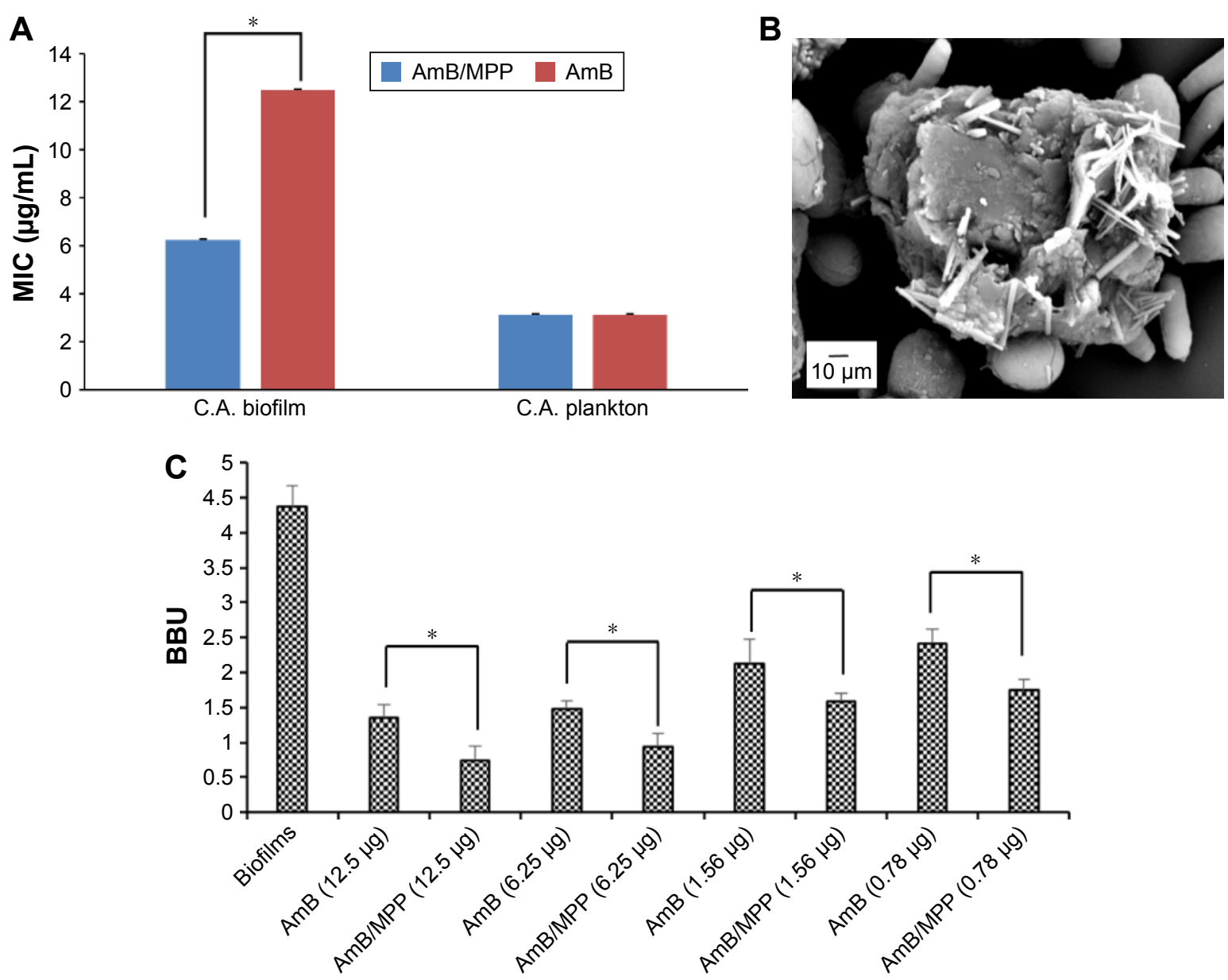

Figure 8 (A) MIC values of AmB/MPP and AmB; (B) observation of Candida albicans mature biofilms under SEM; (C) C. albicans BBU, concentrations of drugs were $\mu$ g/mL. Notes: *Statistical difference at $P<0.05$. All data represent the mean $\pm S D(n=6)$.

Abbreviations: MIC, minimum inhibitory concentration; AmB, amphotericin B; MPP, monomethoxy poly(ethylene glycol)-poly(epsilon-caprolactone)-graft-polyethylenimine; SEM, scanning electron microscope; BBU, biofilm biomass unit; SD, standard deviation; C.A., Candida albicans.

in DMSO is immediately available. Moreover, considering that the mechanism for AmB activity seems to be related to the formation of multimeric drug associations, it could be possible that the drug released from the micelles is partially in conformation with the target interaction.

In this study, C. albicans were incubated for $48 \mathrm{~h}$ in order to form the mature biofilms, which could be verified through the observation under SEM (Figure 8B). From the picture, we could see the extra-polymeric matrix wrapped around the cells. The effects were assessed against $C$. albicans strains using XTT reduction assay that showed a concentrationdependent reduction in biofilm biomass of Candida strains. The increased resistance of sessile cells to antibiotic treatment is mainly due to the biofilm formation. It was obvious that AmB-incorporated micelles have significantly improved the anti-biofilm effect of C. albicans (Figure 8C), which was similar to another report. ${ }^{54}$ To directly quantify the effect of micelles on biofilms, CV staining was used expressed in BBU. The results showed a correlation between the $\mathrm{CV}$ assay and XTT reduction assay. This confirms that AmBloaded micelles were more effective in inhibiting the growth of $C$. albicans biofilms. It may indicate that these micelles presented a superior penetration ability to pass through the extra-polymeric matrix and interact with the membrane of C. albicans. On the other hand, the cation polymer PEI with positive charge had more affinity to the biofilm that showed negative charge on the surface. Fungus would favor the adhesion over the surface of the cation polymer PEI; thus, MPP may influence the adhesion of $C$. albicans on the biotic host surface. Apart from that, there have been many reports that self-aggregated AmB is toxic to both mammalian and fungal cell membranes, while the monomeric form of the drug is toxic to fungal cell membrane only. ${ }^{55,56}$ The favorable result with cell and tissue toxicity of AmB-incorporated micelles appears to be associated with the existence of the drug molecules in the monomeric form in the micelles, whereas 
the antifungal effect did not weaken, suggesting an interesting practical application.

\section{Conclusion}

In this study, we prepared AmB/MPEG-PCL-g-PEI micelles and improved the aqueous solubility of AmB. The micelles were spherical shaped, nano-sized, and stably dispersed with high DL\% (8.60\% $\pm 0.21 \%)$ and EE\% (94.11\% $\pm 1.25 \%)$. DSC assay suggested that AmB was encapsulated into the core-shell structure. The micelles allowed a sustained in vitro release in both normal conditions $(\mathrm{pH} 6.8)$ and $C$. albicans infection pathological conditions ( $\mathrm{pH}$ 5.8) without $\mathrm{pH}$ dependence. Then, the buccal tablets containing AmB/ MPP micelles were produced by direct compression process and evaluated as regards to weight variation, hardness, and friability. In vitro drug release of the buccal tablets was measured in both the USP dissolution apparatus and the dissolution rate test apparatus, which were previously designed for in vitro release assessment to simulate in vivo conditions of the oral cavity. The buccal tablets could sustainably release within $8 \mathrm{~h}$. Regarding safety examination of $\mathrm{AmB} /$ MPP micelles, in vivo histopathological data showed no irritation toward buccal mucosa of the rats in both optical microscopy and ultrastructure observation of the tissues. MTT experiment proved that AmB/MPP micelles reduced the cytotoxicity of AmB. In addition, the toxicity of micelles delivered through the gastrointestinal route was also found to be non-systemic toxicity. Furthermore, the antifungal action of AmB/MPP micelles was evaluated. Although AmB/MPP had no obvious improvement as compared to AmB alone in the antifungal effect of planktonic C. albicans, the micelles significantly enhanced the antifungal activity against the biofilm state of $C$. albicans. Thus, it was concluded that the AmB/MPP micelles could have a superior potential to be developed as a more convenient and effective oral buccal delivery system.

\section{Acknowledgments}

This work was supported by the National Natural Science Foundation of China (No 81470549, No 81402925, and No 81400233) and the Fundamental Research Funds for the Central Universities.

\section{Disclosure}

The authors report no conflicts of interest in this work.

\section{References}

1. Ghannoum MA, Rice LB. Antifungal agents: mode of action, mechanisms of resistance, and correlation of these mechanisms with bacterial resistance. Clin Microbiol Rev. 1999;12(12):501-517.
2. Cassone A, Cauda R. Candida and candidiasis in HIV-infected patients: where commensalism, opportunistic behavior and frank pathogenicity lose their borders. AIDS. 2012;26(12):1457-1472.

3. Kim J, Sudbery P. Candida albicans, a major human fungal pathogen. J Microbiol. 2011;49(2):171-177.

4. Hawser SP, Douglas LJ. Resistance of Candida albicans biofilms to antifungal agents in vitro. Antimicrob Agents Chemother. 1995;39(9): 2128-2131.

5. Olsen I. Biofilm-specific antibiotic tolerance and resistance. Eur J Clin Microbiol. 2015;34(5):877-886.

6. Qasim M, Baipaywad P, Udomluck N, Na D, Park H. Enhanced therapeutic efficacy of lipophilic amphotericin B against Candida albicans with amphiphilic poly(N-isopropylacrylamide) nanogels. Macromol Res. 2014;22(10):1125-1131.

7. Laniado-Laborin R, Cabrales-Vargas MN. Amphotericin B: side effects and toxicity. Rev Iberoam Micol. 2009;26(4):223-227.

8. Pippa N, Mariaki M, Pispas S, Demetzos C. Preparation, development and in vitro release evaluation of amphotericin B-loaded amphiphilic block copolymer vectors. Int J Pharm. 2014;473(1-2):80-86.

9. Gupta U, Agashe HB, Jain NK. Polypropylene imine dendrimer mediated solubility enhancement: effect of $\mathrm{pH}$ and functional groups of hydrophobes. J Pharm Pharm Sci. 2007;10(3):358-367.

10. Bansal KK, Kakde D, Purdie L, et al. New biomaterials from renewable resources - amphiphilic block copolymers from delta-decalactone. Polym Chem. 2015;6(40):7196-7210.

11. Vandermeulen G, Rouxhet L, Arien A, Brewster ME, Preat V. Encapsulation of amphotericin B in poly(ethylene glycol)-block-poly(epsiloncaprolactone-co-trimethylenecarbonate) polymeric micelles. Int $J$ Pharm. 2006;309(1-2):234-240.

12. Legrand P, Romero EA, Cohen BE, Bolard J. Effects of aggregation and solvent on the toxicity of amphotericin B to human erythrocytes. Antimicrob Agents Chemother. 1992;36(11):2518-2522.

13. Barwicz J, Christian S, Gruda I. Effects of the aggregation state of amphotericin B on its toxicity to mice. Antimicrob Agents Chemother. 1992;36(10):2310-2315.

14. Lemke A, Kiderlen AF, Kayser O. Amphotericin B. Appl Microbiol Biot. 2005;68(2):151-162.

15. Chen YC, Su CY, Jhan HJ, Ho HO, Sheu MT. Physical characterization and in vivo pharmacokinetic study of self-assembling amphotericin B-loaded lecithin-based mixed polymeric micelles. Int J Nanomedicine. 2015;10:7265-7274.

16. Adams ML, Lavasanifar A, Kwon GS. Amphiphilic block copolymers for drug delivery. J Pharm Sci. 2003;92(7):1343-1355.

17. Gaucher G, Satturwar P, Jones MC, Furtos A, Leroux JC. Polymeric micelles for oral drug delivery. Eur J Pharm Biopharm. 2010;76(2): $147-158$.

18. Wang K, Liu TT, Lin R, et al. Preparation and in vitro release of buccal tablets of naringenin-loaded MPEG-PCL nanoparticles. RSC Adv. 2014;4(64):33672-33679.

19. Wiegand C, Bauer M, Frischmuth T, Fischer D, Hipler UC. Evaluation of the antifungal effect of polyethylenimine (PEI) by laser nephelometry. Mycoses. 2009;52(5):427-427.

20. Wiegand C, Bauer M, Frischmuth T, Fischer D, Hipler UC. Evaluation of the antifungal effect of polyethylenimine (PEI) using microplate laser nephelometry (MLN). J Dtsch Dermatol Ges. 2011;9(1):225-225.

21. Wiegand C, Bauer M, Fischer D, Hipler UC. Determination of the antifungal effect of polyethylenimine (PEI) by microplate laser nephelometry in vitro. Mycoses. 2012;55(4):257-257.

22. Soncini-Júnior G, Franchetti SMM, Marconato JC. Architecture and relevance of several strongly adhered biofilms over a polyester imide (PEI) surface. Braz J Microbiol. 2003;34(34):105-107.

23. Shi S, Zhu XC, Guo QF, et al. Self-assembled mPEG-PCL-g-PEI micelles for simultaneous codelivery of chemotherapeutic drugs and DNA: synthesis and characterization in vitro. Int J Nanomedicine. 2012; 7(9):1749-1759.

24. Hao JS, Heng PWS. Buccal delivery systems. Drug Dev Ind Pharm. 2003;29(8):821-832. 
25. Zhang H, Zhang J, Streisand JB. Oral mucosal drug delivery - clinical pharmacokinetics and therapeutic applications. Clin Pharmacokinet. 2002;41(9):661-680.

26. Reddy PC, Chaitanya KSC, Rao YM. A review on bioadhesive buccal drug delivery systems: current status of formulation and evaluation methods. Daru. 2011;19(6):385-403.

27. Mrsny RJ. Oral drug delivery research in Europe. J Control Release. 2012;161(2):247-253.

28. Dabholkar RD, Sawant RM, Mongayt DA, Devarajan PV, Torchilin VP. Polyethylene glycol-phosphatidylethanolamine conjugate (PEGPE)-based mixed micelles: some properties, loading with paclitaxel, and modulation of P-glycoprotein-mediated efflux. Int J Pharm. 2006;315(1-2):148-157.

29. Casa DM, Carraro TC, de Camargo LEA, Dalmolin LF, Khalil NM, Mainardes RM. Poly(L-lactide) nanoparticles reduce amphotericin B cytotoxicity and maintain its in vitro antifungal activity. $J$ Nanosci Nanotechnol. 2015;15(1):848-854.

30. Lavasanifar A, Samuel J, Sattari S, Kwon GS. Block copolymer micelles for the encapsulation and delivery of amphotericin B. Pharm Res. 2002;19(4):418-422.

31. Diaz IL, Parra C, Linarez M, Perez LD. Design of micelle nanocontainers based on PDMAEMA-b-PCL-b-PDMAEMA triblock copolymers for the encapsulation of amphotericin B. AAPS PharmSciTech. 2015;16(5):1069-1078.

32. Tang XL, Liang Y, Zhu YQ, et al. Anti-transferrin receptor-modified amphotericin B-loaded PLA-PEG nanoparticles cure Candidal meningitis and reduce drug toxicity. Int J Nanomedicine. 2015;10: 6227-6241.

33. Duan Y, Cai X, Du H, Zhai G. Novel in situ gel systems based on P123/TPGS mixed micelles and gellan gum for ophthalmic delivery of curcumin. Colloids Surf B Biointerfaces. 2015;128:322-330.

34. Butt AM, Amin MCIM, Katas H, Sarisuta N, Witoonsaridsilp W, Benjakul R. In vitro characterization of pluronic F127 and D-alphatocopheryl polyethylene glycol 1000 succinate mixed micelles as nanocarriers for targeted anticancer-drug delivery. J Nanomater. 2012; 2012:11.

35. Zhang W, Shi Y, Chen YZ, Hao JG, Sha XY, Fang XL. The potential of pluronic polymeric micelles encapsulated with paclitaxel for the treatment of melanoma using subcutaneous and pulmonary metastatic mice models. Biomaterials. 2011;32(25):5934-5944.

36. Keegan GM, Smart JD, Ingram MJ, Barnes LM, Burnett GR, Rees GD. Chitosan microparticles for the controlled delivery of fluoride. J Dent. 2012;40(3):229-240.

37. Schneyer LH, Levin LK. Rate of secretion by individual salivary gland pairs of man under conditions of reduced exogenous stimulation. $J$ Appl Physiol. 1955;7(5):508-512.

38. Jain V, Gupta A, Pawar VK, et al. Chitosan-assisted immunotherapy for intervention of experimental leishmaniasis via amphotericin B-loaded solid lipid nanoparticles. Appl Biochem Biotechnol. 2014; 174(4):1309-1330.

39. Gangadhar KN, Adhikari K, Srichana T. Synthesis and evaluation of sodium deoxycholate sulfate as a lipid drug carrier to enhance the solubility, stability and safety of an amphotericin B inhalation formulation. Int J Pharm. 2014;471(1-2):430-438.
40. O'Toole GA, Kolter R. Initiation of biofilm formation in Pseudomonas fluorescens WCS365 proceeds via multiple, convergent signalling pathways: a genetic analysis. Mol Microbiol. 1998;28(3):449-461.

41. Asthana S, Gupta PK, Jaiswal AK, Dube A, Chourasia MK. Targeted chemotherapy of visceral leishmaniasis by lactoferrin-appended amphotericin B-loaded nanoreservoir: in vitro and in vivo studies. Nanomedicine. 2015;10(7):1093-1109.

42. Kim YT, Shin BK, Garripelli VK, et al. A thermosensitive vaginal gel formulation with HPgammaCD for the $\mathrm{pH}$-dependent release and solubilization of amphotericin B. Eur J Pharm Sci. 2010;41(2):399-406.

43. Aframian DJ, Davidowitz T, Benoliel R. The distribution of oral mucosal $\mathrm{pH}$ values in healthy saliva secretors. Oral Dis. 2006;12(4):420-423.

44. Xia BF, Yang Z, Zhou HY, et al. Development of a novel oral cavity compartmental absorption and transit model for sublingual administration: illustration with Zolpidem. AAPS J. 2015;17(3):631-642.

45. Lalla RV, Bensadoun RJ. Miconazole mucoadhesive tablet for oropharyngeal candidiasis. Expert Rev Anti Infect Ther. 2011;9(1):13-17.

46. Torrado JJ, Espada R, Ballesteros MP, Torrado-Santiago S. Amphotericin B formulations and drug targeting. J Pharm Sci. 2008;97(7):2405-2425.

47. Van de Ven H, Paulussen C, Feijens PB, et al. PLGA nanoparticles and nanosuspensions with amphotericin $\mathrm{B}$ : potent in vitro and in vivo alternatives to Fungizone and AmBisome. J Control Release. 2012; 161(3):795-803

48. Burgess BL, Cavigiolio G, Fannucchi MV, Illek B, Forte TM, Oda MN. A phospholipid-apolipoprotein A-I nanoparticle containing amphotericin B as a drug delivery platform with cell membrane protective properties. Int J Pharm. 2010;399(1-2):148-155.

49. Thomas P, Hecker J, Faunt J, Fenech M. Buccal micronucleus cytome biomarkers may be associated with Alzheimer's disease. Mutagenesis. 2007;22(6):371-379.

50. Gandhi RB, Robinson JR. Oral cavity as a site for bioadhesive drugdelivery. Adv Drug Deliv Rev. 1994;13(1-2):43-74.

51. Dowty ME, Knuth KE, Irons BK, Robinson JR. Transport of thyrotropin-releasing-hormone in rabbit buccal mucosa in vitro. Pharm Res. 1992;9(9):1113-1122.

52. Squier CA, Wertz PW. Structure and function of the oral mucosa and implications for drug delivery. Drugs Pharm Sci. 1996;74:1-26.

53. Rathbone MJ, Tucker IG. Mechanisms, barriers and pathways of oral mucosal drug permeation. Adv Drug Deliv Rev. 1993;12(1-2):41-60.

54. Randhawa MA, Gondal MA, Al-Zahrani AH, Rashid SG, Ali A. Synthesis, morphology and antifungal activity of nano-particulated amphotericin-B, ketoconazole and thymoquinone against Candida albicans yeasts and Candida biofilm. J Environ Sci Health A Tox Hazard Subst Environ Eng. 2015;50(2):119-124.

55. Bolard J, Legrand P, Heitz F, Cybulska B. One-sided action of amphotericin B on cholesterol-containing membranes is determined by its self-association in the medium. Biochemistry. 1991;30(23): 5707-5715.

56. Brajtburg J, Elberg S, Travis SJ, Kobayashi GS. Treatment of murine candidiasis and cryptococcosis with amphotericin B incorporated into egg lecithin-bile salt mixed micelles. Antimicrob Agents Chemother. 1994; 38(2):294-299. 


\section{Supplementary materials Evaluation of buccal tablets}

Tablet weight variation

Twenty tablets were weighed by an electronic scale (AY 120; Shimadzu Co., Ltd., Kyoto, Japan), and the average weight of the tablets was calculated.

\section{Hardness}

Hardness was measured using a hardness tester(YD-1; Shanghai Develop Machinery Co., Limited, Shanghai, China). Six tablets from three formulations were randomly tested. The mean and standard deviation values were obtained.

\section{Friability}

Friability was determined through a friability tester (CJY300B; Shanghai Hongyi, Shanghai, China). After removing the superfluous powder, 60 preweighed tablets were placed in the tester and rotated $4 \mathrm{~min}$ at $25 \mathrm{rpm}$. Then, the powder of the tablets was gently loosened with a blower and reweighed. The percentage loss was calculated as follows:

$$
\% \text { loss }=\frac{\text { Initial weight }- \text { Final weight }}{\text { Initial weight }} \times 100
$$

\section{LC-MS analysis}

LC 20AD HPLC system (Shimadzu Co., Ltd.) was coupled to a triple-quadrupole tandem mass spectrometer (API3200; AB SCIEX, Foster City, CA, USA) in electron spray ionization mode to monitor positive molecular ions $[\mathrm{M}+\mathrm{H}]^{+}$. High-performance liquid chromatography (HPLC) analysis was performed on a Dikma C18 column $(250 \times 4.6 \mathrm{~mm}$, $5 \mu \mathrm{m})$ at $30^{\circ} \mathrm{C}$ with the injection volume of $20 \mu \mathrm{L}$. The mobile phase (acetonitrile: $10 \mathrm{mmol}$ ammonium acetate aqueous solution $=40: 60,1 \mathrm{~mL} / \mathrm{min}$ ) from the HPLC column was delivered directly into the mass spectrometer with parameters as follows: optimized ion spray voltage $5,000 \mathrm{~V}$, optimized temperature $600^{\circ} \mathrm{C}$, and $\mathrm{N} 2$ pressure GS1 $=40$ psi, GS2 $=15$ psi. The operating conditions were as follows: collision energy, declustering potential, entrance potential, and collision cell exit potentials were 129, 41, 9, and $38 \mathrm{~V}$ for $\mathrm{AmB}$. The mass transition used for $\mathrm{AmB}$ was m/z 922.608/81 Da.

\section{A}

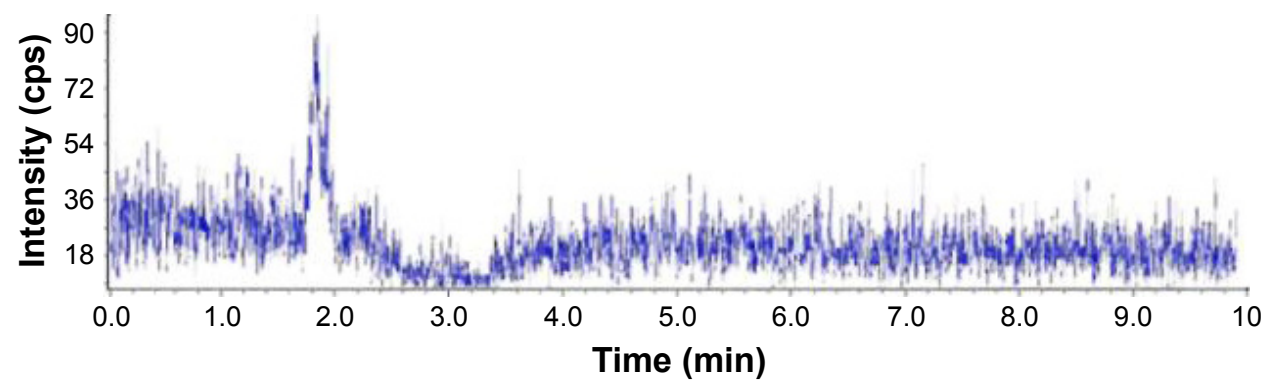

B

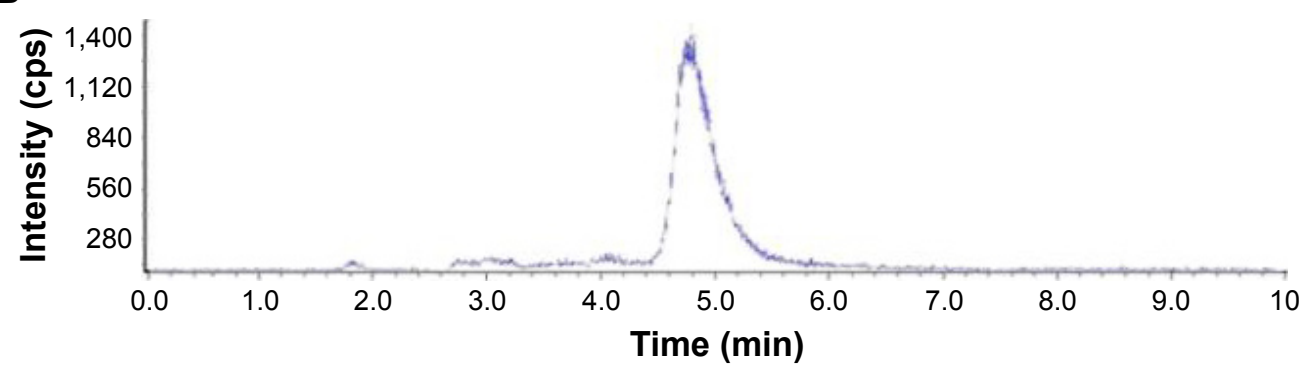

Figure SI (Continued) 
C

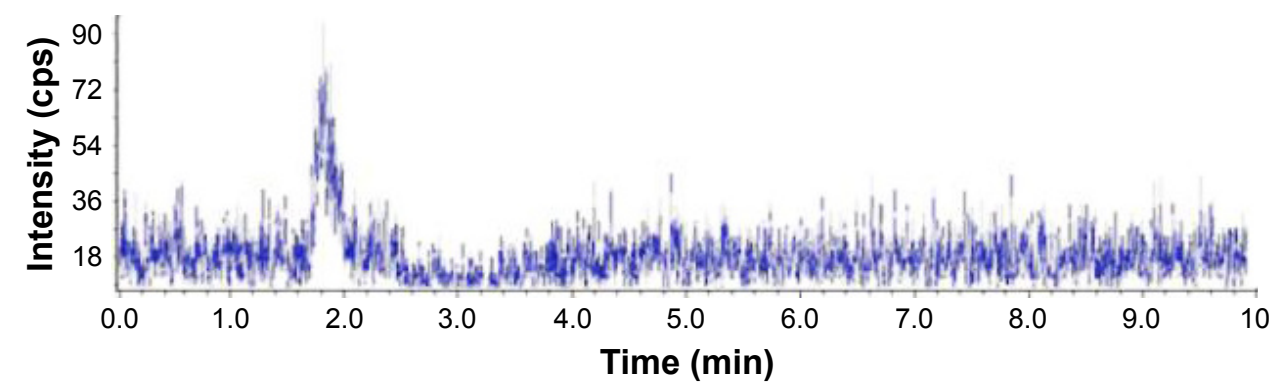

Figure SI LC-MS spectrum for the toxicity of AmB/MPP micelles through (A) gastrointestinal tract, (B) blank plasma, and (C) AmB in blank plasma, after $8 \mathrm{~h}$ dosing of a plasma sample.

Abbreviations: AmB, amphotericin B; MPP, monomethoxy poly(ethylene glycol)-poly(epsilon-caprolactone)-graft-polyethylenimine; LC-MS, liquid chromatography-mass spectrometry.

\section{Publish your work in this journal}

The International Journal of Nanomedicine is an international, peerreviewed journal focusing on the application of nanotechnology in diagnostics, therapeutics, and drug delivery systems throughout the biomedical field. This journal is indexed on PubMed Central, MedLine, CAS, SciSearch $®$, Current Contents $\AA /$ Clinical Medicine,
Journal Citation Reports/Science Edition, EMBase, Scopus and the Elsevier Bibliographic databases. The manuscript management system is completely online and includes a very quick and fair peer-review system, which is all easy to use. Visit http://www.dovepress.com/ testimonials.php to read real quotes from published authors.

Submit your manuscript here: http://www.dovepress.com/international-journal-of-nanomedicine-journal 